\title{
A Tracked Mobile Robotic Lab for Monitoring the Plants Volume and Health
}

\author{
M Bietresato, G Carabin, \\ D D'Auria, R Gallo, G Ristorto, \\ F Mazzetto and R Vidoni \\ Faculty of Science and Technology \\ UniBZ - University of Bolzano \\ piazza Università, 39100 Bolzano (I) \\ Email: marco.bietresato, giovanni.carabin, \\ daniela.dauria, raimondo.gallo, gianluca.ristorto, \\ fabrizio.mazzetto,renato.vidoni@unibz.it
}

\author{
A Gasparetto and L Scalera \\ DPIA - Dipartimento Politecnico di \\ Ingegneria e Architettura \\ UniUD - Università di Udine \\ via delle Scienze, Udine (I) \\ Email: lor.scalera@gmail.com, gasparetto@uniud.it
}

\begin{abstract}
Precision agriculture has been increasingly recognized for its potential ability to improve agricultural productivity, reduce production cost, and minimize damage to the environment.

In this work, the current stage of our research in developing a mobile platform equipped with different sensors for orchard monitoring and sensing is presented. In particular, the mobile platform is conceived to monitor and assess both the geometric and volumetric conditions as well as the health state of the canopy. To do so, different sensors have been integrated and effective data-processing algorithms implemented for a reliable crop monitoring. Experimental tests have been performed allowing to obtain both a precise volume reconstruction of several plants and an NDVI mapping suitable for vegetation state evaluations.
\end{abstract}

\section{INTRODUCTION}

One of the most important trends in crop production is to apply precision agriculture (PA) methodologies for different targets such as site-specific application of fertilizer and onfarm management of production systems. In this contest, PA, also called site-specific agriculture, can be seen as the application of information technology to agriculture allowing studying fields on a finer resolution and providing opportunities for increasing the efficiency and decreasing the production costs. New technology is indeed used or redesigned to make agriculture more efficient. For instance, the use of Global Positioning Systems (GPS) allows to have GPS-computer guided harvestors and geo-referenced site-specific operations such as soil sample and crop yield data collection, aerial imagery, etc. The geo-referenced information can then be used to influence management decisions to maintain an optimal vegetativeproductive balance on the plant and obtain sustainable yields of quality.

Focusing on the direct observation of the tree canopy to obtain agronomic information, i.e. the crop monitoring, an integrated-mechatronic approach that puts together mobile (semi-)autonomous robotic platforms, proper new and effective (vision) sensors within ad-hoc developed data-processing and control algorithms, can be the key for designing future smart and effective solutions.

\section{A. Agricultural Robotics}

Since the 1980s, the application of robotics in agriculture has been seen as an interesting field of research [1], even if only with the availability of new and low cost sensors and controllers together with the development of the mobile robots research field, effective results and success technologies have been realized.

Introduce robots or semi-automated machines in different field activities, such as planting, weeding, crop and environmental monitoring, fruit picking, etc., would allow to implement the PA template and reach important levels of production together with a correct and sustainable soil exploitation. Then, their use will need an automatic operational monitoring system, possibly integrating the acquired data coming from their applications into an information system framework tailored to farm requirements [2].

Locomotion, stability and balancing is surely a challenging feature both for travelling on uneven terrains and for reducing the measurement problems on sensors due to the oscillations that the terrain could create on the mobile platform. On flat lands, wheeled configurations are surely suitable but on rough and/or unexplored terrains the wheels performance can significantly drop. However, different robotic architectures for future use as robotic prototypes to complex farm activities and operating as implement-carriers are available: tracked, three-wheeled, four-wheeled conventional, four-wheeled with an articulated chassis, legged/walking and hybrid leg-wheel systems [3], [4], [5], [6]. Some of them are already used in human-driven agricultural machines such as small- and largesized tracked, articulated and conventional tractors and selfpropelled operating machines. Nevertheless, the optimal robot configuration depends strongly on the working environment [1]. Investigation is still required because, when considering the possibility of automating the navigation of a vehicle by introducing sophisticated driving systems and monitoring activity, it is necessary to identify the configuration that better responds to the activity needs and constraints. 


\section{B. Methods and devices for crop sensing and measurement}

The recent availability of affordable sensors and electronic systems is facilitating the intensive measurements procedures by replacing traditional manual measurements. Currently, almost all the plant characteristics, e.g. canopy height, width and volume, leaf area index, have an associated mechatronic sensors and technology to assess it allowing to increase the accuracy of the measurements. Thus, huge amounts of measured data, suitably processed, help decision making in crop management.

In particular, geometry, e.g. canopy height, width and volume, and structural parameters, e.g. leaf area index, canopy porosity, are important parameters due to their direct influence on the plants behavior and on the knowledge and prediction of the vigor and quality of the produced crop [7]. Canopy volume is an important data since it allows to combine the width, height, geometric shape and structure of trees[8]; usually, this volume measurement required costly and timeexpensive manual-made measurements of the so-called "TreeRow-Volume" (i.e., it is the envelope volume) by calculating the average crop's height and width. In literature, the sensorization and automation of the measurement procedure have been treated with several sensor-based approaches based either on the use of electromagnetic radiation, e.g. digital photography, photogrammetry, and stereoscopy techniques as well as LiDAR (Light Detection And Ranging) sensors [9], here exploited, or on the use of ultrasonic waves.

Regarding the LiDAR sensor, it estimates the distance apart of the object of interest by performing sweeps in a plane (2D) or in space (3D). Commonly, a so-called mobile terrestrial laser scanner (MTLS) is proposed: a 2D LiDAR sensor mounted on a vehicle moving along the rows of trees in an orchard [10]. Other works report, for instance, on a LiDAR sensor mounted on a ground tripod used for $3 \mathrm{D}$ volumetric modeling of a grapevine [11], or on a ground fixed LiDAR sensor to 3D model the tree skeletons [12], [13], [14]. Often, a LiDAR sensor and a GPS receiver are mounted on a same chassis for 3D reconstruction of orchards. Performance relies on the precision of the GPS and on the position of the LiDAR sensor [15], [16], [17], [18].

The plants health is another important data that has to be estimated. To do so, optical crop-sensing systems that use light sensors, e.g. GreenSeeker and OptRx, are proposed since the red light absorption is directly related to the plants health. In particular, vegetation indexes based on plant biomass and nitrogen content are computed; the Normalized Difference Vegetative Index (NDVI)[19] is the most common one and, for instance, the here chosen OptRX sensor gives as output Vegetative Index (VI) such as NDVI and NDRE (Normalized Difference Red Edge Index); the OptRX is an active sensor that illuminates the plant canopy ba means of a polychromatic light source allowing it to distinguish its own light signal from that of surrounding ambient light.

\section{Aims}

In this work, the current stage of our research related to the development of a crop monitoring mobile semi-autonomous terrestrial vehicle based on a combined use of optical sensors is presented. In particular, in Section II, the chosen mobile platform is described together with the chosen equipment and algorithms used. Then, in Section III, experimental tests and results are reported and discussed while, in Section V, conclusions and future work are listed.

\section{THE MOBILE LAB}

The mobile vehicle to be used for plants sensing throughout has to show some basic technical requirements: (a) be able to move nimbly within the orchards, travel off-road and perform turning manoeuvres even in steep hill-sides; (b) be easily transportable to limit the costs; (c) allow to lodge on it the individuated crop monitoring sensors by means of an ad-hoc structure.

The chosen mobile system is a modified tracked bins-carrier (the NEO Alpin by Windegger S.r.l., Lana, Bolzano, Italy). It is an electric-driven and wireless remote-controlled system with very compact dimensions (length $\mathrm{x}$ width: $1.14 \mathrm{x} 1.12$ $\mathrm{m})$ and a high carrying capacity $(500 \mathrm{~kg})$ with respect to its mass $(250 \mathrm{~kg})$, see Fig. 1a. As shown in Fig. 1b, it has been equipped with a vertical adjustable frame made of steel and aluminium tubular beams for housing and fixing the different sensors, e.g. LiDAR, NDVI. Different widths and heights for housing the sensors can be chosen to experiment many configurations and eventually adapt the system to the height and inter-row distance of the orchard under evaluation.

All the sensors are powered by means of the mobile platform battery. The system architecture is made by a central unit connected with an acquisition board; thanks to this, the different sensors are connected and powered over the proper LAN, USB and RS232 buses.

The central unit runs a real-time LabView program, which task is acquiring and properly storing data from the different sensors, described in the next section. At the end of the acquisition, the raw data are post-processed by means of different algorithms developed to create the volume and healthy maps, implemented in Matlab $^{T M}$.

\section{A. Sensors}

The main materials used to develop the overall system are a set of co-operating sensors. More in details, 2 LiDAR sensors vertically aligned at two different heights and 6 OptRx crop sensors vertically aligned and at three different heights (3 on the left and 3 on the right side) scanning the same targets have been installed on the NEO. In such a manner, given the three measurements, the idea is to try to reconstruct the vegetative state of the plant. The top view of the canopy, here not considered, could represent an important measurement and, in the Author's idea, it will be integrated by means of a air-drone.

Specifically, two SICK LMS111 LiDAR active sensors, thus neither dependent on sunlight nor on clear sky, were installed 


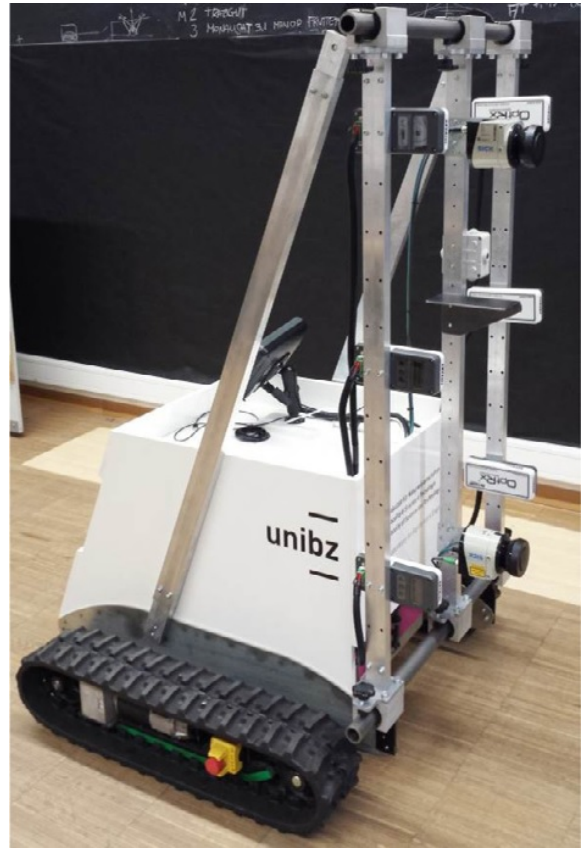

(a)

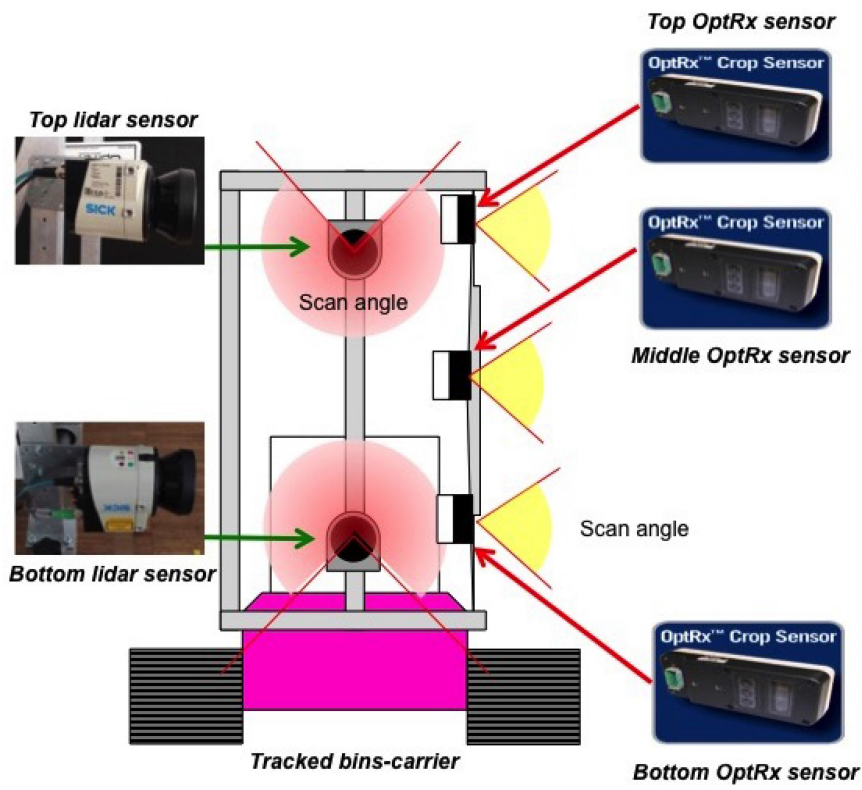

(b)

Fig. 1: NEO mobile robot equipped with sensors. a) lateral view; b) frontal view and sensors placement.

on an adjustable vertical frame, the one in the higher position upside-down, i.e. with the blind scan angle of 90deg on the top, vice versa for the lower sensor (see Fig. 1b). The LMS111 sensor falls within the short-range devices, has a maximum radius of 20 meters and working-angle of $270^{\circ}$ (see Table I for the device main technical characteristics).

TABLE I: LiDAR SICK LMS111 characteristics

\begin{tabular}{c|c}
\hline Features & LMS111 \\
\hline Max Range & $20 \mathrm{~m} / 18 \mathrm{~m}$ \\
Scanning Angle & Max $270^{\circ}$ \\
Angular resolution & $0.5^{\circ} / 0.25^{\circ}$ adjustable \\
Scanning frequency & $50 / 25 \mathrm{~Hz}$ \\
Response time & $20 \mathrm{~ms} / 40 \mathrm{~ms}$ \\
Error (stat) & $12 \mathrm{~mm}$ typ. \\
Sender & Pulsed laser diode \\
Light source & Infrared $(905 \mathrm{~nm})$ \\
Operating temp & $-30^{\circ} \mathrm{C}$ to $50^{\circ} \mathrm{C}$ \\
\hline
\end{tabular}

The six OptRx Crop Sensors, available from AgLeader, work by shining light on the crop canopy and reading the light reflected back to determine the crop health, also known as Vegetative Index (VI). They are able to record and measure real time information such as the Vegetative Indexes NDVI (Normalized Difference Vegetation Index) and NDRE (Normalized Difference Red Edge Index) with a scanning angle of 45 - $10 \mathrm{deg}$ and a measurement range of 0.25-2 m.

Moreover, in order to track the robot position both a sonar sensor and a RTK GPS have been installed on the system. The former allows to have the position data of the mobile lab in terms of distance from a movable target (e.g., a vertical panel) inner a lab, where the GPS is not properly working; the latter provides GPS position accuracy to within 1 centimeter thanks to a separate base station located within approximately $8 \mathrm{~km}$ of the mobile unit. In order to have information also on the mobile robot orientation on uneven terrains, the mobile robot has been equipped with a LMRK 10 AHRS Inertial Measurement Unit from Gladiator Technologies that allows to measure angular position (e.g. Euler angles), velocity and acceleration, magnetic field and temperature.

\section{B. Data Processing}

The two LiDAR sensors have been installed with the higher sensor upside-down, i.e. with the blind scan angle of 90deg on the top, while the lower in the normal configuration. In such a way, a vision system composed by two LiDAR sensors aligned vertically has been developed and, scanning the same targets, a sort of new "lateral-linear-stereoscopic LiDAR-based vision" has been realized. Since each LiDAR scan as output gives a series of points representing the profile of the scanned bodies in a vertical plane normal to the robot travelling direction, by means of an ad-hoc developed algorithm, it is possible to approximate any scanned profile with a poly-line that merges the acquired points. Finally, the objects area and volume can be computed [20], [21].

The plants health estimation is made by means of the OptRX sensors. Indeed, by processing the acquired normalized difference vegetation index (NDVI), a normalized ratio of the NIR (near infra-red) and red bands [22], it is possible to understand the differences in vegetative development among plant groups (e.g. high NDVI values are recognized as dense 
vegetation). The optical-data collected can then be processed in order to obtain the NDVI maps that show differences in vegetative development among plant group and be merged and superimposed with the previous volume estimation and maps elaborations. Moreover, by correlating the vegetation thickness (e.g. dividing it in ranges) with the NDVI index, it is possible to create additional new simplified maps that allow to better highlight different vegetation healthy and unhealthy regions, e.g. where foliage is sparse.

\section{EXPERIMENTAL TESTS}

The developed (semi-)autonomous Terrestrial Vehicle, programmed to travel at a constant speed along a straight line, e.g. an orchard row, was used to characterize the trees in Fig. 2 , chosen for their well known shape and height. Thus, in

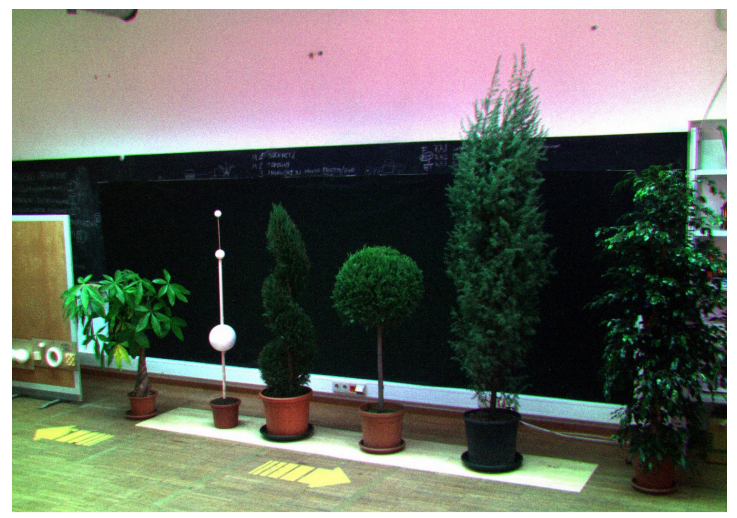

(a)

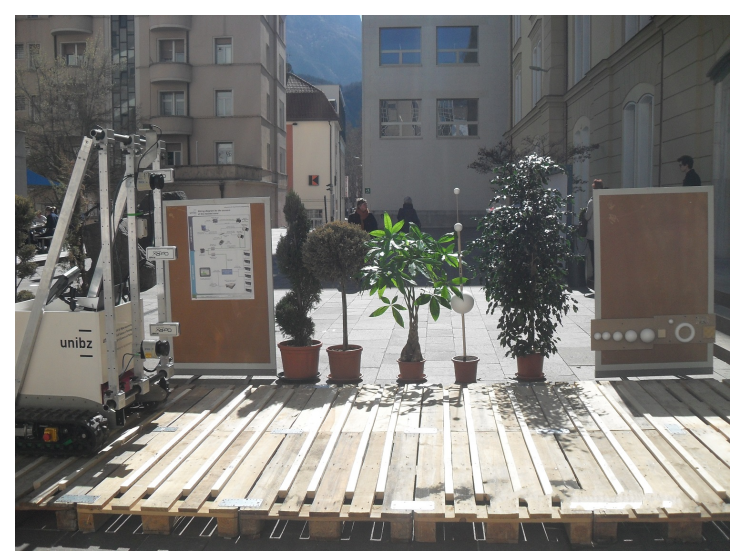

(b)

Fig. 2: Experimental layout of the scanned plants: (a) indoor and (b) outdoor scenario

experimental tests we evaluated:

- Cupressocyparis Leylandii Spiral, height $160 \mathrm{~cm}$;

- Cupressocyparis Leylandii Ball, height $140 \mathrm{~cm}$;

- Cupressocyparis Leylandii Pon Pon, height 160 cm;

- Juniperus virginiana, height $240 \mathrm{~cm}$;

- Pachira, height $110 \mathrm{~cm}$;

- Ficus benjamina, height $160 \mathrm{~cm}$.
Then, a fake plant made of polystyrene balls mounted on a plastic rod, the second from the left in Fig. 2a, with a well known geometry and volume has been added together with some reference panels made of wood, see $2 b$ before and after the plants. The panels have been added to the scenario to define and recognize the plane defined by the row under test and normal to the terrain. An experimental activity indoor and outdoor aimed at acquiring processing data for canopy estimation and monitoring in different scenarios has been performed, Fig. 2. Firstly, an indoor environment, i.e. Fig. 2a, has been experimentally evaluated and, thanks to the encouraging results, an outdoor scenario, i.e. Fig. 2b, has been set-up for testing the mobile system and exploiting the GPS sensor.

\section{RESULTS AND DISCUSSION}

The combined use of LiDAR and NDVI sensors has been evaluated to get on one side accurate information about the geometric shapes and the vegetative states of the plants and on the other side the canopy reconstruction of the plant line.

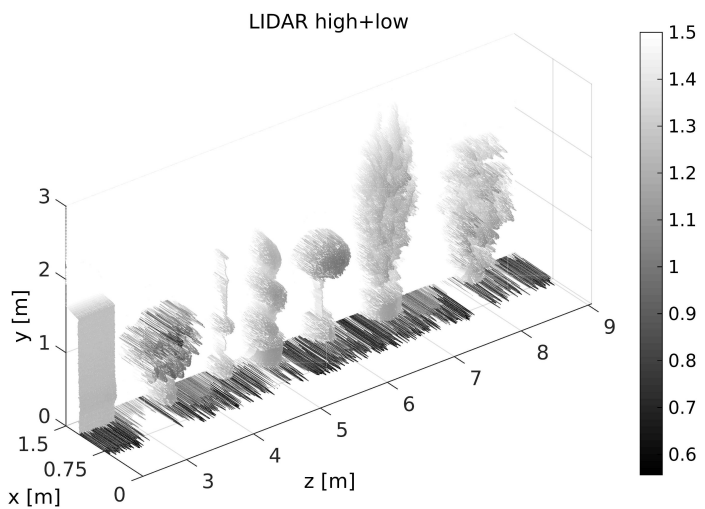

(a)

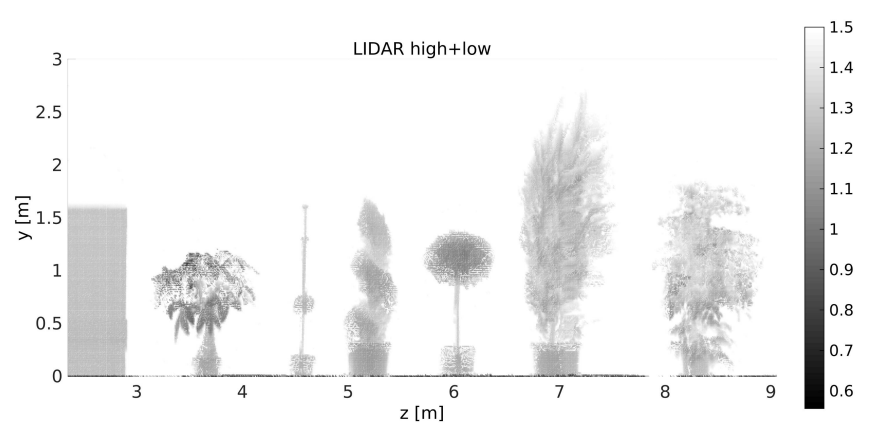

(b)

Fig. 3: Volume-LiDAR resulting representation of the scanned plants with the lateral-linear-stereoscopic LiDAR-based vision: (a) 3D and (b) frontal view

Firstly, the data obtained with the LiDAR elaborations have been processed using Matlab by means of the method previously explained [20]. The resulting scan of an indoor 


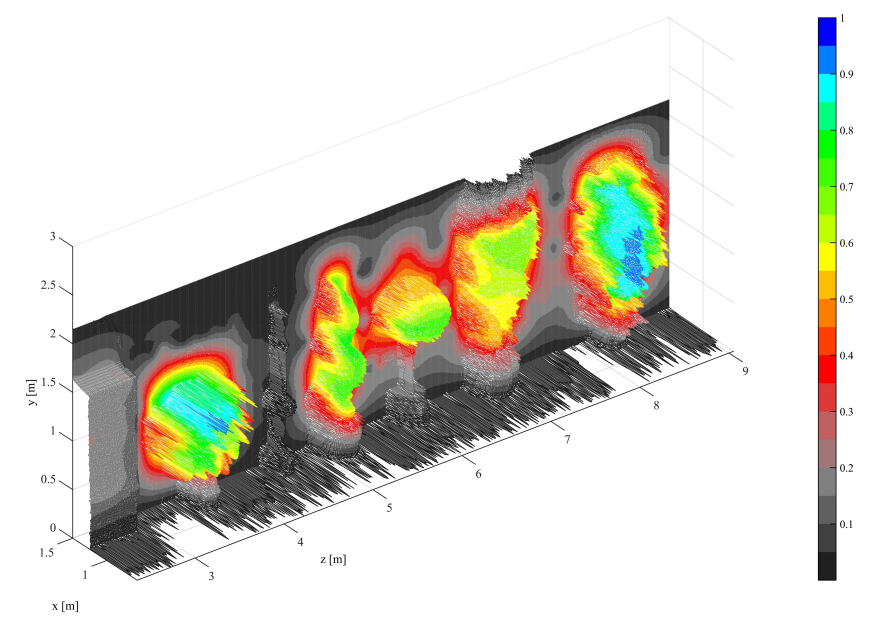

(a)

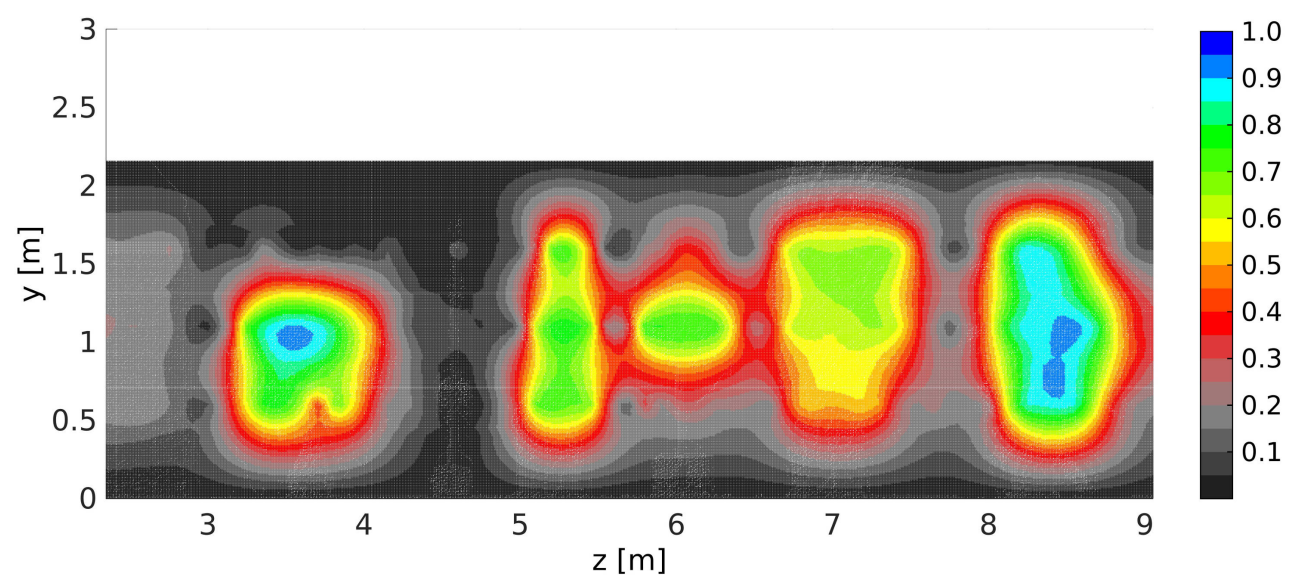

(b)

Fig. 4: Volume-LiDAR results merged with the OptRX data of the scanned plants: (a) 3D and (b) frontal view

acquisition is presented in Fig. 3. Results show a very good representation of the plants volumes. In Fig. 3a the 3D view is presented while in Fig. $3 b$ the plot is projected in two dimensions. As can be seen, thanks to the lateral-linear-stereoscopic LiDAR-based vision, the objects and plants contours and volumes are very well estimated (see [21] for more details on the algorithm used and its performance); indeed, on the left side the recognized reference panel allows to define the zerocoordinate of the row and to tune the measured data. Secondly, after an overlapping phase, the distance data have been postprocessed and meshed with the data form the OptRx showing interesting results, Fig. 4. It can be seen how the NDVI maps reconstruct the vegetative state of the plant line of interest in a suitable manner.

As can be appreciated in Fig. 5, where a portion of the final representation is magnified, the fake plant is easily recognized, i.e. black - very low NDVI values, while the vegetation is present with the correct values and colours, i.e. vegetative state. Moreover, also the flowerpots, being not vegetation, show a very low vegetation index, i.e. near to zero, as well as the trunk parts. In order to define and choose the thresholds for recognizing different vegetative states, different choices have to be done according to the orchard plants, e.g. apple, pear, etc.

Current activities are devoted to the outdoor tests of the mobile system and fast data processing of the acquired data in particular on a real orchard for assessing the performances in a real environment.

\section{CONCLUSION AND FUTURE WORK}

Inner the precision agriculture and proximal sensing research area, this work presents the current research stage of Authors' activities on the design, development and implementation of a (semi-)autonomous mobile robotic platform equipped with state-of-the-art sensors for the volume, vegetation and health estimation of plants inner an orchard. After the 


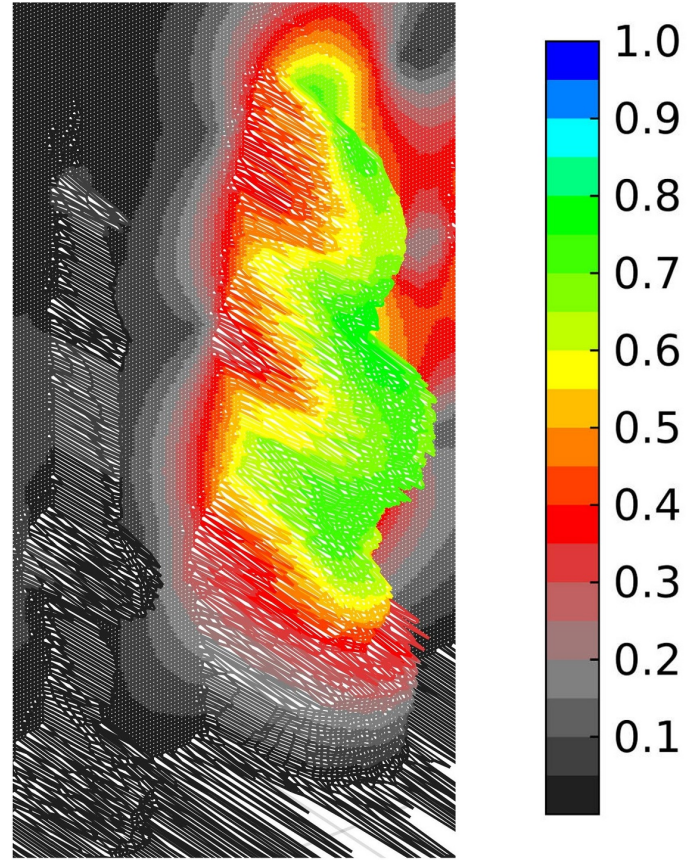

Fig. 5: Particular of the merged volume-LiDAR + OptRX results

description of the chosen tracked mobile platform, the main vision and implemented sensors chosen and installed on the system have been presented together with the data-processing procedure and developed algorithms.

The system has been tested in a semi structured environment showing interesting results and performance allowing to start outdoor intensive tests for the tuning of the algorithms and proper evaluation of both the hardware and data-logger requirements, and on the robustness of the measurements to terrain and atmosphere non-ideal conditions.

The evaluation of some specific indices to significantly improve the data fusion and analysis efficiency, together with the integration in management framework for assessing in a fast and automatic manner the plant evolution over time will be part of the future activities of the project.

\section{ACKNOWLEDGMENT}

The research leading to these results has been supported by the Monalisa project, which was funded by the Autonomous Province of Bozen-Bolzano.

\section{REFERENCES}

[1] J. Billingsley, A. Visala, and M. Dunn, Springer Handbook of Robotics. Berlin, Heidelberg: Springer Berlin Heidelberg, 2008, ch. Robotics in Agriculture and Forestry, pp. 1065-1077.

[2] A. Calcante and F. Mazzetto, "Design, development and evaluation of a wireless system for the automatic identification of implements," Computers and Electronics in Agriculture, vol. 101, pp. 118 - 127, 2014.

[3] R. Siegwart, I. Nourbakhsh, and D. Scaramuzza, Introduction to autonomous mobile robots (2nd ed.), T. M. Press, Ed. Cambridge, Massachusetts, USA: Massachusetts Institute of Technology, 2011.
[4] S. Yaghoubi, N. Akbarzadeh, S. Bazargani, S. Bazargani, M. Bamizan, and M. Asl, "Autonomous robots for agricultural tasks and farm assignment and future trends in agro-robots," International Journal of Mechanical and Mechatronics Engineering IJMME-IJENS, vol. 13, no. 3, pp. $1-6,2013$.

[5] C. Wright, A. Buchan, B. Brown, J. Geist, M. Schwerin, D. Rollinson, M. Tesch, and H. Choset, "Design and architecture of the unified modular snake robot," in Robotics and Automation (ICRA), 2012 IEEE International Conference on, May 2012, pp. 4347-4354.

[6] R. Vidoni, M. Bietresato, A. Gasparetto, and F. Mazzetto, "Evaluation and stability comparison of different vehicle configurations for robotic agricultural operations on side-slopes," Biosystems Engineering, vol. 129, pp. $197-211,2015$.

[7] K. Lee, R. Ehsani, and W. Castle, "A laser scanning system for estimating wind velocity reduction through tree windbreaks," Journal of Computers and Electronics in Agriculture, vol. 73, pp. 1-6, 2010.

[8] R. Sanz, J. R. Rosell, J. Lorens, E. Gil, and S. Planas, "Relationship between tree row lidar-volume and leaf area density for fruit orchards and vineyards obtained with a lidar $3 \mathrm{~d}$ dynamic measurement system," Agricultural and Forest Meteorology, 2013.

[9] J. R. Rosell and R. Sanz, "A review of methods and applications of the geometric characterization of tree crops in agricultural activities," Computers and Electronics in Agriculture, vol. 81, pp. 124-141, 2012.

[10] J. Rossel Polo, R. Sanz, J. Lorens, J. Arnó, A. Escolá, M. RibesDasi, J. Masip, F. Camp, F. Gracia, F. Solannes, T. Pallejà, L. Val, S. Planas, E. Gil, and J. Palacin, "A tractor-mounted scanning lidar for the non-destructive measurement of vegetative volume and surface area of treerow plantations: A comparison with conventional destructive measurements," Biosystems Engineering, vol. 102, pp. 128-134, 2009.

[11] K. Keightley and G. Bawden, "3d volumetric modeling of grapevine biomass using tripod lidar," Comput. Electron. Agr., vol. 74, pp. 305$312,2010$.

[12] A. Bucksch and S. Fleck, "Automated detection of branch dimensions in woody skeletons of leafless fruit tree canopies," SilviLaser 2009, Oct 2009.

[13] P. Raumonen, M. Kaasalainen, M. Kerblom, S. Kaasalainen, H. Kaartinen, M. Vastaranta, M. Holopainen, M. Disney, and P. Lewis, "Fast automatic precision tree models from terrestrial laser scanner data," Remote Sensing, vol. 5, pp. 491-520, 2013.

[14] J.-F. Coöte, J.-L. Widlowski, R. Fournier, and M. Verstraete, "The structural and radiative consistency of three-dimensional tree reconstructions from terrestrial lidar," Remote Sensing of Environment, vol. 113, pp. 1067-1081, 2009.

[15] P. Walklate, J. Cross, G. Richardson, R. Murray, and D. Baker, "Comparison of different spray volume deposition models using lidar measurements of apple orchards," Biosys. Eng., vol. 82, pp. 253- 267, 2002.

[16] F. Auat Cheein and J. Guivant, "Slam-based incremental convex hull processing approach for treetop volume estimation," Computers and Electronics in Agriculture, vol. 102, pp. 19-30, 2014.

[17] C. Jaeger-Hansen and K. Dhring, "Electric agricultural robot with multilayer-control," International Conference of Agricultural Engineering, 2012.

[18] J. Rosell, J. Llorens, R. Sanz, J. Arnó, M. Ribes-Dasi, J. Masip, A. Escolá, F. Camp, F. Solanelles, F. Gracia, E. Gil, L. Val, S. Planas, and J. Palacin, "Obtaining the three-dimensional structure of tree orchards from remote $2 \mathrm{~d}$ terrestrial lidar scanning," Agriculture and Forest Meteorology, vol. 149, pp. 1505-1515, 2009.

[19] F. Povh and G. de Paula, W. dos Anjos, "Optical sensors applied in agricultural crops," Optical Sensors - New Developments and Practical Applications, 2014.

[20] M. Bietresato, G. Carabin, R. Vidoni, A. Gasparetto, and F. Mazzetto, "Evaluation of a lidar-based 3d-stereoscopic vision system for cropmonitoring applications," Computers and Electronics in Agriculture, vol. 124, pp. $1-13,2016$.

[21] M. Bietresato, R. Vidoni, A. Gasparetto, and F. Mazzetto, "Design and first tests of a vision system on a tele-operated vehicle for monitoring the canopy vigour status in orchards," 2015, pp. 126-130.

[22] J. Rouse, R. Haas, and D. Schell, J.A. Deering, "Monitoring vegetation systems in the great plains with erts," Third Earth Resources Technology Satellite Syposium, vol. 1, pp. 309-317, 1974. 